\title{
Multi-objective Particle Swarm Optimization Based Image Watermarking Scheme
}

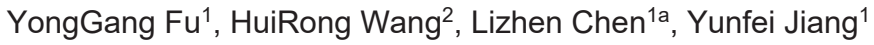 \\ ${ }^{1}$ College of computer engineering, Jimei University, Xiamen, 360121, China \\ ${ }^{2}$ College of Chinese Literature, Jimei University, Xiamen, 361021, China
}

\begin{abstract}
A novel image watermarking scheme based on the statistics of the blocked DCT coefficients. The watermark is embedded into the middle frequency of those DCT coefficients by modulating the number of those positive and negative coefficients. In order to achieve better robustness and imperceptibility, multi-objective particle swarm optimization (MPSO) has been used in the watermark embedding and extracting procedure. The particle swarm optimization is applied to obtain optimum multiple scaling factors and the embedding strength. The experimental results show that the proposed scheme has significant improvement in term of imperceptibility and robustness under various general attacks.
\end{abstract}

\section{Introductions}

Computer and networks have been a significant way to exchange information in this era. Various digital multimedia products are spread on the Internet. Nowadays, people can copy, alter, and transmit multimedia properties easily, including texts, images, audios, videos, and so on. Modern information technology brings not only convenient and cheap digital transmission services, but also various challenges. One of the most important is how to protect the properties of the legal owner [1]. In this case, digital watermarking is proposed, which means hiding information or anticounterfeit mark into the host image to protect copyright. It will be flourishing in the next decades with the urgent market demand. It is the digital watermarking technology that may be utilized as a supplement for cryptography encryption and scrambling technology [2].

During the last decade, many robust image watermarking algorithms have been proposed, which can be divided into three categories according to the domain what the watermark can be embedded: spatial domain watermarking, transform domain and hybrid domain watermarking. The spatial domain watermarking algorithms [3, 4]mainly embed the watermark by modulating the image pixels or altering its gray values directly, such as the least significant bit (LSB) and so on. These algorithms are fragile to manipulations, but they have little computation work and can be easily implemented. The transform domain watermarking schemes are mainly concentrated on some signal transforms such as DCT(Discrete Cosine Transform) [5, 6], DFT(Discrete Fourier Transform) [7] and DWT(Discrete Wavelet Transform) [8-10], etc. The watermark is embedded into the host by modifying some or all frequency domain coefficients, especially middle frequency coefficients. These methods have better robustness and can resist common attacks [11-13]. The hybrid domain can employ the advantages in two or more domain. The typical scheme is based on two transform domains. The first transform may have some good characteristics to some common attacks, and the second transform can be robust to geometric attacks. The hybrid domain algorithms can achieve good performance, but they are complex in implementation. It may be a future development direction because computer performance is strengthening on calculation. Now, most of the proposed watermarking schemes are based on the transformation domain. In this paper, the proposed algorithm can effectively resist some geometrical attacks.

The paper is organized as follows. In section II, the particle swarm optimization is reviewed. Section III describes the proposed watermarking scheme in detail. The experimental results are shown in section VI. And section VII conclude the paper.

\section{The particle swarm optimization}

The particle swarm optimization is a meta-heuristic swarm based algorithm widely used in parameter optimization, which is proposed by Kennedy and Eberhart [14]. The particle swarm optimization mimics the simplified social models such as fish schooling and bird flocking. A swarm is defined as a set of mobile agents that collectively carry out problem solving in a distributed manner. Each agent is called one particle. In a swarm, each particle keeps track of their own attributes.

\footnotetext{
${ }^{\mathrm{a}}$ Corresponding author: f_rock@163.com
} 
Initially, the position $x_{(i, j)}(k)$ and the velocity $v_{(i, j)}(k)$ of each particle $p$ are randomly created. Each particle represents a candidate solution to optimize problem by calculate its fitness. The fitness of each particle is evaluated by an objective function. During iteration, the best location visited by each particle is kept as the local best position $P_{\text {best }(i, j)}$, while the best location visited by all particles is kept as the global best position $G_{\text {best }(i, j)}$.

Therefore, a new population is created based on a preceding one and the particles are updated by the following equations:

$$
\left\{\begin{aligned}
V_{(i, j)}(k+1)= & \omega V_{(i, j)}(k)+c_{1} r_{1}\left[P_{\text {best } t i, j)}(k)-L_{(i, j)}(k)\right] \\
& +c_{2} r_{2}\left[G_{\text {best }(i, j)}(k)-L_{(i, j)}(k)\right] \\
L_{(i, j)}(k+1)= & L_{(i, j)}(k)+V_{(i, j)}(k+1)
\end{aligned}\right.
$$

Where those parameters can be depicted as follows: $(i, j)$ : index of the position in the particle; $k=1,2, \ldots \mathrm{P}$, is the iteration number; $v_{(i, j)}(k)$ : velocity of the particle in the swarm at the position index $(i, j) ; L_{(i, j)}(k)$ is the current position of the particle at the position index $(i, j)$; $r_{1}$ and $r_{2}$ are random values uniformly distributed in range $[0,1] ; \quad c_{1}$ and $c_{2}$ are positive acceleration coefficients, which control the influence of $P_{\text {best }(i, j)}$ and $G_{\text {best }(i, j)} ; \omega$ : inertia weight, usually with in $0.8 \leq \omega \leq 1.4$.

The detail procedure of PSO algorithm can be depicted as follows:

Algorithm 1. Particle Swarm Optimization(PSO)

1) Create a population, $P$ of random particles.

2) For each particle calculate the fitness value and find the local best $\mathrm{P}_{\text {best. }}$.

3) Calculate the global best, $\mathrm{G}_{\text {best }}$ for the swarm.

4) Update each particle's velocity and position using Eq.1.

5) If termination condition is not met, goto step 2).

\section{Proposed watermark embedding and extracting scheme}

The block DCT coefficients can be divided into DC coefficient and AC coefficients. The DC coefficients of $m$ th block can be expressed $\mathrm{F}_{\mathrm{m}}(0,0)$ and the rest may be divided into low, middle and high frequency coefficients. Since tiny modification of the DC or low frequency coefficients may degrade image subjective quality, embedding watermark into the DC or low frequency coefficients should not be adopted. The watermark information embedded into high frequency coefficients can be easily removed during the general image

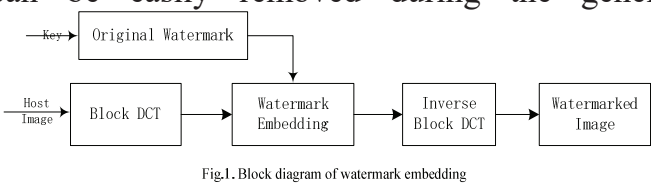

processing. So the middle frequency coefficients may be a better choice according to the necessity of compromise between robustness and invisibility. In this paper, the watermark is embedded into the middle frequency coefficients. Watermark embedding and extracting block

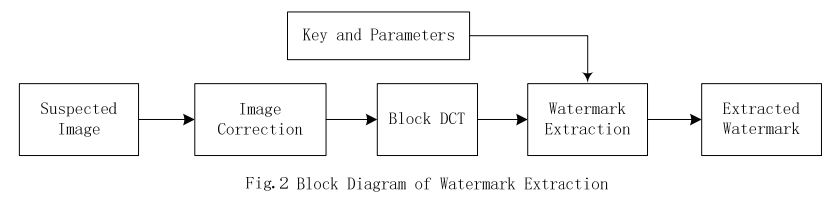

diagrams are shown in Fig. 1 and Fig. 2 respectively:

Firstly, the host image is divided into disjoint blocks and then transformed to frequency domain by block-DCT. And mid-frequency DCT coefficients are selected for watermark embedding. The Zigzag scanning of $8 \times 8$ coefficients and embedding positions can be shown in Fig. 3:

Since slight modification of the $1^{\text {st }}, 2^{\text {nd }}$ and $3^{\text {rd }}$ coefficients may affect the image quality serious, middle frequency coefficients are selected for watermark embedding. The proposed watermark embedding and extraction procedure can be depicted as following steps:

1) Watermark embedding procedure

(1) Divide the original image into $8 \times 8$ blocks denoted as $f_{m}(i, j)$, and then they are transformed to DCT domain coefficients $F_{m}(u, v)$. The logo watermark is denoted as watermark $(k)$;

(2) $F_{m}(u, v)$ is scanned into $F_{m}(s)$ with zigzag scanning, and those coefficients between $k_{1}^{\text {th }}$ and $k_{2}^{\text {th }}$ are selected. The positive and negative numbers among these coefficients can be denoted as $T p(i, j)$ and $\operatorname{Tn}(i, j)$, respectively.

(3) Modify the coefficients and getting modified coefficients $F_{m}{ }^{\prime}(u, v)$ as following:

(i) If watermark $(k)=1, T p(i, j)=\alpha(p, q) T n(i, j)$

(ii) If watermark $(k)=0, T n(i, j)=\alpha(p, q) T p(i, j)$

Those selected coefficients are modified by changing the sign of the least coefficients in ascending order of their absolute values until the numbers of positive or

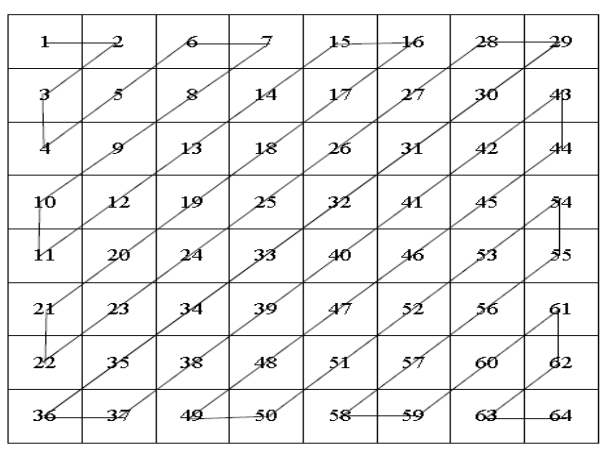

Fig.3. The zigzag scanning of the $8 \times 8$ DCT negative coefficients to be $T p$ or $T n$, respectively.

(4) Every block $F_{m}{ }^{\prime}(u, v)$ is transformed by inverse block DCT and merged into the watermarked image $I^{\prime}$. 
Where the parameters are as follows: $0 \leq i, j, u, v \leq 8,0 \leq k_{1} \leq k_{2} \leq 63, m=M * N / 64$ , $\mathrm{M}^{*} \mathrm{~N}$ is the original host image size.

In a word, every watermark bit can be embedded in each block. The total watermark capacity may be 1024 bits.

After analyzing the characteristics of middle frequency coefficients, it can be found that the absolute values are very close, so we introduce an embedding strength factor, which can not only adjust the compromise between robustness and invisibility, but also eliminate rounding error from the function uint8. This error may affect the watermark extraction.

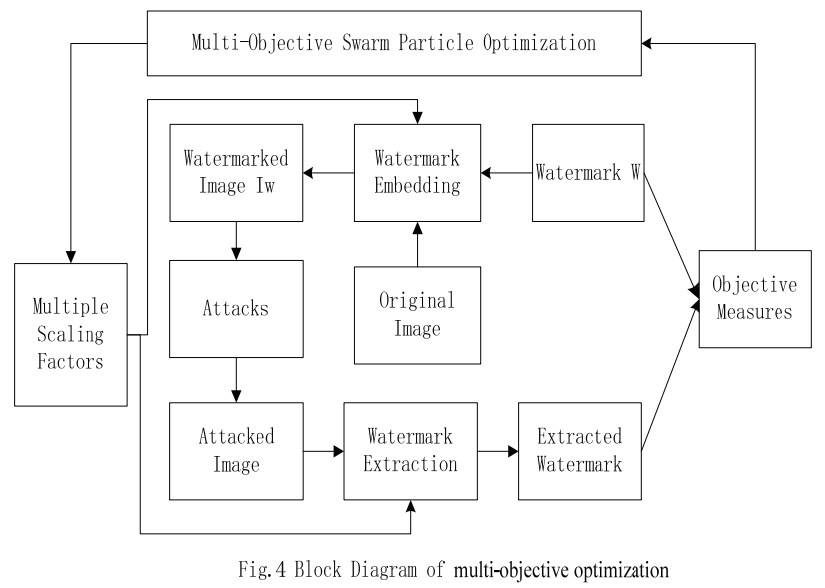

2) Determining multiple scaling factors:

A parameter $\alpha(p, q)$ which is used to control the tradeoff between the imperceptibility and robustness, generally used as scaling factor, should be decided firstly. If the scaling factor is different in each watermark bit to be embedded, $\alpha$ can be a matrix, i.e. multiple scaling factors. To determine the optimal values of these multiple scaling factors can be viewed as an optimization problem. Multi-objective particle swarm optimization may be applied.

The block diagram of multi-objective optimization is a closed-loop control system. The system input is multiple scaling factors and objective measure as system output. This measure is calculated from original image $I$, watermarked image $I_{w}$, watermark $W$ and the $(\mathrm{T}+1)$ extracted watermarks.

The steps for applying a multi-objective particle swarm optimization into the proposed watermarking scheme are enumerated.

3) Watermark extraction procedure:

(a) Divide the suspected image into $8 \times 8$ blocks and perform the DCT transform on each block. The $m$-th block coefficients are denoted as $F_{m}{ }^{\prime}(u, v)$;

(b) Scan $F_{m}(u, v)$ into $F_{m}(s)$ with zigzag scanning, and select those coefficients between the $k_{1} t h$ and $k_{2} t h$. The positive and negative numbers of them are counted as $T p^{\prime}(i, j)$ and $T n^{\prime}(i, j)$, respectively.

(1) If $T p^{\prime}(i, j) \geq \alpha(p, q) T n^{\prime}(i, j)$, ewatermark $(k)=1$
(2) If $T p{ }^{\prime}(i, j)<\alpha(p, q) T n^{\prime}(i, j)$, ewatermark $(k)=0$

(c) Merge those extracted bits ewatermark into logo image.

Where the parameters are as follows: $0 \leq i, j, u, v \leq 8,0 \leq k_{1} \leq k_{2} \leq 63, m=M * N / 64$ , $\mathrm{M}^{*} \mathrm{~N}$ is the original host image size.

\section{Experimental results}

In order to evaluate the performance of the proposed method, some experimental results are done on MATLAB Ra2008. The experiments are carried out on several images including "Lena", "Pepper", "Plane", "Baboon", which represent different texture complexity. Due to the limitation of space, here only demonstrate the experimental results on Lena with size $256 \times 256$ Lena gray image. Fig. 5 shows the original image, watermarked image, logo watermark and the extracted watermarked.

\subsection{Objective image visual quality evaluation}

Image visual quality evaluation methods can mainly be divided into two categories which are subjective ones and objective ones. The subjective ones are the methods of grading subjective quality or video expert group, etc. The objective one are the methods of the mean square error (MSE), the signal-to-noise ratio (SNR), the peak signalto-noise ratio (PSNR), the structure similarity (SSIM), and other methods based on human visual characteristics. Here mainly uses the mean square error (MSE) and the peak signal-to-noise ratio (PSNR) to measure the image quality.

Suppose the host image $f(i, j)$ being the gray value of L-level image whose size is $N \times N, f^{\prime}(i, j)$ is the gray value of the watermarked image, and $0 \leq i, j \leq N-1$, MSE and PSNR are defined as follows:

$$
\begin{aligned}
& M S E=\frac{1}{N \times N} \sum_{i=0}^{N} \sum_{j=0}^{N}\left|f(i, j)-f^{\prime}(i, j)\right|^{2} \\
& P S N R=10 \log _{10} \frac{(L-1)^{2}}{M S E}
\end{aligned}
$$

The smaller MSE is, the distortion is less and the PSNR is larger; the larger MSE is, the distortion is more serious and the PSNR is smaller.

\subsection{Extracted watermark quality evaluation}

The original watermark and the extracted watermark can be denoted as watermark $(i, j)$ and ewatermark $(i, j)$ respectively, whose size is $N / 8 \times N / 8$. The bit error ratio (BER) is used to evaluate the quality of extracted watermark. The BER can be defined as follows: 


$$
B E R=\frac{64}{N \times N} \sum_{i, j} \operatorname{ewatermark}(i, j) \neq \operatorname{watermark}(i, j)
$$

2. H.Berghal and L.O.Gorman, Protecting ownership rights through digital watermarking[J]. IEEE Computer, 1996. 29(7): p. 67-72.

Where $\sum$. is the sum on all the bit errors, and $0 \leq i, j \leq N / 8$.

\subsection{Experimental results}

We choose five $256 \times 256$ gray images (Lena, Cameraman, Couple, Peppers, and Barbara) to do the simulation experiments. The block size is $8 \times 8$. The results are shown in Table 2 . The watermark embedded is 1024 bits. The strengths are set to $d=k_{1}-k_{2}+1=28-7+1=22$. The PSNR and the BER result of watermark extraction non-using MPSO and using MPSO method are shown in Table 1.

Table 1 Experimental result of Proposed Scheme

\begin{tabular}{lllll}
\hline Attacks & \multicolumn{3}{l}{ PSNR (dB) } & $\begin{array}{l}\text { BER (Bit Error } \\
\text { Rate }\end{array}$ \\
\cline { 2 - 5 } & $\begin{array}{l}\text { Non- } \\
\text { PSO }\end{array}$ & $\begin{array}{l}\text { Using } \\
\text { PSO }\end{array}$ & $\begin{array}{l}\text { Non- } \\
\text { PSO }\end{array}$ & $\begin{array}{l}\text { Using } \\
\text { PSO }\end{array}$ \\
\hline JPEG (75\%) & 29.472 & 33.4573 & 0.772 & 0.8573 \\
\hline JPEG (85\%) & 32.5443 & 34.6574 & 0.8443 & 0.8874 \\
\hline $\begin{array}{l}\text { Gaussian noise } \\
(2 \%)\end{array}$ & 31.5785 & 32.6342 & 0.8785 & 0.9342 \\
\hline Scaling $(25 \%)$ & 31.3235 & 33.5343 & 0.8235 & 0.9343 \\
\hline $\begin{array}{l}\text { Salt\&pepper } \\
(2 \%)\end{array}$ & 30.5241 & 32.7645 & 0.8241 & 0.9545 \\
\hline Noisy $(2 \%)$ & 31.5363 & 32.6465 & 0.8563 & 0.9465 \\
\hline $\begin{array}{l}\text { Cropping } \\
(10 \%)\end{array}$ & 29.4232 & 31.5433 & 0.8632 & 0.9533 \\
\hline Blurring $(4 \times 4)$ & 30.3423 & 33.4554 & 0.8423 & 0.9154 \\
\hline $\begin{array}{l}\text { Sharpening } \\
(4 \times 4)\end{array}$ & 32.2136 & 34.5765 & 0.9136 & 0.9365 \\
\hline Rotation $\left(3^{\circ}\right)$ & 29.5712 & 31.6357 & 0.8712 & 0.9457 \\
\hline
\end{tabular}

\section{Conclusions}

A novel block DCT based image watermarking scheme is proposed in this paper. MPSO is used to optimize the embedding scaling factors to achieve a good compromise between the invisibility and robustness. The experimental results show that the proposed method based on MPSO is robust to general attacks and resilience to some geometric attacks.

\section{Acknowledgments}

This work was partly supported by the Soft Science Foundation of Fujian Province of China (No. 2016R0067), and Special Foundation of Fujian Province Educational Program (No.Z816239).

\section{References}

1. I.J. Cox, et al., Secure Spread Spectrum Watermarking for Multimedia[J]. IEEE transactions on image processing, 1997. 6(12): p. 1673-1687.
3. C.-K. Chan and Cheng, L.M., Hiding data in images by simple LSB substitution[J]. Pattern Recognition, 2004. 37: p. 469-474.

4. D.P. Mukherjee, Maitra, S., and Acton, S.T., spatial domain digital watermarking of multimedia objects for buyer authentication[J]. IEEE transactions on multimedia, 2004. 6(1): p. 1-15.

5. S. Alyammahi, et al. A new multiple watermarking scheme for copyright protection and image authentication. in IEEE International Midwest Symposium on circuits and systems. 2016. Abu Dhabi, UAE.

6. C. Li and Qin, Z. A blind digital image watermarking algorithm based on DCT. in IET International conference on smart and sustainable city. 2013.

7. V. Solachidis and Pitas, L., Circularly symmetric watermark embedding in 2-D DFT domain[J]. IEEE transactions on image processing, 2001. 10(11): p. 1741-1753.

8. K. Zebbiche and Khelifi, F., Efficient wavelet-based perceptual watermark masking for robust fingerprint image watermarking[J]. IET Image Process, 2014. $8(1)$ : p. 23-32.

9. C.M.Namratha and S.Kareemulla. Multi image watermarking using Lagrangian support vector regression. in IEEE International Conference on recent trends in electronics information communication technology. 2016. India.

10. C. Mingzhi, et al., A combined DWT and DCT watermarking scheme optimized using genetic algorithm[J]. Journal of Multimedia, 2013. 8(3): p. 299-305.

11. E.Esen and A.A.Alatan, Robust video data hiding using forbidden zone data hiding and selective embedding $[\mathrm{J}]$. IEEE transactions on circuits and systems for video technology, 2011. 21(8): p. 11301137.

12. Y. Fu, Robust oblivious image watermarking scheme based on coefficient relation[J]. Optik - International Journal for Light and Electron Optics, 2013. 124(6): p.

13. L. An, et al., Robust lossless data hiding using clustering and statistical quantity histogram[J]. Neurocomputing, 2012. 77: p. 1-11.

14. J. Kennedy and Eberhart, R.C., Particle swarm optimization[D], in Proceedings of IEEE International conference on neural networks. 1995. p. 1942-1948. 517-521. 